\title{
Nephro Fachtagung Ulm Nachlese 2016 und Ausblick auf 2017
}

Die staatlich anerkannte Nephrologische Weiterbildungsstätte Ulm (www.wbs-ulm. de) veranstaltete in Kooperation mit der AfnP e.V. (www.afnp.de) und dem Georg Thieme Verlag zum 6. Mal die Nephro Fachtagung Ulm. Wir bedanken uns für die sehr positiven Rückmeldungen der Teilnehmer.

Die Nephro Fachtagung Ulm hat sich als erfolgreiche und interessante Veranstaltung im süddeutschen Raum etabliert.

\section{Veranstaltung 2016}

Der Rückzug der Industrie aus den pflegerischen Fortbildungen hat die Teilnehmer verärgert, da direkte Gespräche mit Industrievertretern nur noch auf pflegerischen Veranstaltungen möglich sind und nicht mehr im Alltag auf der Dialyse. Wir bedanken uns bei den folgenden Firmen:

- Arbeitsgemeinschaft für nephrologisches Personal e.V. (AfnP)

- Bundesarbeitsgemeinschaft Nephrologische Pflege (BANP)

- Bioscientia

- Fresenius Medical Care

- Georg Thieme Verlag KG

- Staatlich anerkannte Nephrologische Weiterbildungsstätte Ulm (WBS-Ulm)

Unser Programm gestaltet der Programmbeirat und wählt Referenten aus, die nicht im finanziellen Interessenkonflikt stehen. Aus diesem Grund haben wir uns schon vor Jahren für die Zusammenarbeit mit dem Georg Thieme Verlag entschieden und dies nicht bereut. Wichtig ist für uns die neutrale Information bei unseren Veranstaltungen. Die Vorträge können von den Teilnehmern auf www.nephro-ulm. de heruntergeladen werden.

Eine logistische Herausforderung war die kurzfristige Verlegung des ursprünglich geplanten Tagungsorts Kornhaus Ulm ins Hotel Seligweiler im Herbst 2015, was uns gut gelungen ist. In Seligweiler lässt sich noch einiges optimieren, aber aus der Erfahrung und der Rückmeldung der Teilnehmer können wir uns verbessern. In der Tagungsgebühr inbegriffen waren 2 Kaffeepausen und ein Mittagessen (Auswahl aus 4 Gerichten mit Getränk). Es wurde so viel diskutiert, dass die Pausenplanung nicht eingehalten werden konnte. Das werden wir 2017 entsprechend einplanen und die Pausen verlängern.

Der Tagungsort Hotel Seligweiler $(12 \mathrm{~km}$ von der Innenstadt Ulm entfernt mit sehr guter Autobahnanbindung und kostenfreien Parkplätzen) kam bei den meisten Teilnehmern gut an. Der eingerichtete Shuttle-Service vom Bahnhof Ulm zum Tagungsort wurde von den Teilnehmern erst zu spät bemerkt. Das werden wir auf der Homepage besser kommunizieren. Ca. 2 Drittel der Teilnehmer wünschten sich eine gemeinsame Abendveranstaltung, ca. ein Drittel möchte den Abend frei gestalten. Wir werden dies in der Planung für 2017 berücksichtigen und umsetzten. Im nächsten Jahr bieten wir am Freitagabend eine Fahrt in die Innenstadt Ulm und einen Abholservice gegen 22:30 Uhr an.

Die Teilnehmer kamen bundesweit aus Deutschland und aus dem deutschsprachigen Raum. Die Frühregistrierung wurde von über 90 Teilnehmern genutzt und auch die Möglichkeit, kurzfristig die Veranstaltung zu besuchen. Besonders gefragt waren die Tageskarten am Samstag. Durchweg gab es ein sehr positives Fazit der Teilnehmer. An dieser Stelle möchten wir uns für die ausgefüllten Evaluierungsbögen bedanken (Auszug aus der Auswer-

Tab. 1 Auszug aus der Teilnehmerevaluierung.

\begin{tabular}{|c|c|c|c|c|c|}
\hline Wie zufrieden waren Sie mit ... & ++ & + & 0 & - & -- \\
\hline ... der Organisation vor der Veranstaltung? & $50 \%$ & $40 \%$ & $9 \%$ & & $1 \%$ \\
\hline ... dem Veranstaltungsort? & $36 \%$ & $40 \%$ & $20 \%$ & $4 \%$ & \\
\hline ... der Wartezeit an der Kasse? & $61 \%$ & $31 \%$ & $8 \%$ & & \\
\hline ... dem organisatorischen Ablauf der Tagung? & $48 \%$ & $41 \%$ & $11 \%$ & & \\
\hline ... der Themenauswahl? & $36 \%$ & $48 \%$ & $16 \%$ & & \\
\hline ... der Erfüllung Ihrer persönlichen Erwartung? & $22 \%$ & $56 \%$ & $16 \%$ & $6 \%$ & \\
\hline ... der Qualität der Vorträge? & $29 \%$ & $64 \%$ & $6 \%$ & $1 \%$ & \\
\hline
\end{tabular}

tung siehe Tab. 1). Wir werden versuchen, die vielen Anregungen und Themenwünsche für 2017 umzusetzen. Die Hitliste der Vorträge 2016 findet sich im Folgenden:

- Platz 1: „Der vergessene Patient - Demenz in der Akutversorgung“, Michael Metzger, München. Die AfnP und die WBS-Ulm bieten zusammen mit Herrn Metzger ein Tagesseminar am Dienstag, den 25.10.2016, in Ulm an (Anmeldung ist noch möglich).

- Platz 2: „AV-Innovation: die endovaskuläre Fistelanlage“, Julian Rieck, Therapy Development, TVA Medical

- Platz 3: „Humangenetik“, Prof. Carsten Bergmann, Ingelheim, und „Dialyse \& Soziales: Sozialrechtliche Aspekte der chronischen Nierenerkrankung inkl. Lebendnierenspende“, Dipl.-Sozialpädagogin Nicole Scherhag, Saulheim. Die AfnP und die WBS- Ulm bieten zusammen mit Frau Scherhag ein Tagesseminar am Samstag, den 29.10.2016, in Ulm an (Anmeldung ist noch möglich).

- Platz 4: "Phosphathaushalt", PD Lars Rothermund, Ulm

- Platz 5: „Wie erreiche ich mein Team? Möglichkeiten der Mitarbeitermotivation“, Dipl.-Pflegewirtin (FH) Martina Kaufmann, Oberammergau

- Platz 6: „Ökonomie und Versorgungsqualität - Passt das zusammen?“, Dr. Thomas Weinreich, VillingenSchwenningen, „Exotische Shunts Transplantation der Shuntvene, Kollateralenbypass etc.“, Dr. Franziska Frizen, Wiesbaden, und „Gerontonephrologie - keine Zukunftsvision sondern bereits Alltag! Praxisrelevante Besonderheiten in der Versorgung betagter nephrologischer Patienten“, Dr. Stefan Nunnenkamp, Richard Kaltenhauser; Pfarrkirchen

\section{Veranstaltung 2017}

Das Hotel Seligweiler wird 2016 ausgebaut: So werden 60 Zimmer sowie 4 Tagungsräume angebaut und der Wellnessbereich erweitert sowie modernisiert. Damit wird der Tagungsort 2017 noch attraktiver.

Schon heute möchten wir Sie zur nächsten Nephro Fachtagung Ulm am 10. und 11.03.2017 einladen (www.nephro-ulm. de). Die Planungen laufen bereits. Viele Anregungen und Themenwünsche aus Ihren Rückmeldungen werden wir 2017 umsetzen und ein neues Thema ist in der Vorbereitung. Lassen Sie sich überraschen! Marion Bundschu, Ulm 